\title{
Expectation and repetition effects in searching for featural singletons in very brief displays
}

\author{
LIQIANG HUANG and HAROLD PASHLER \\ University of California-San Diego, La Jolla, California
}

\begin{abstract}
We studied the effects of expectation and repetition on searching for singletons in very brief displays. In Experiment 1, we found that when a given feature defined the singleton for a whole blockso that in every trial the subject could expect a particular target feature-search accuracy was significantly higher than when the feature setting was randomly redetermined from trial to trial. However, an unexpected repetition triggered almost no advantage. In Experiment 2, we found no advantage for expected alternation. Experiments 3 and 4 demonstrated that there is little or no advantage for perception in conditions allowing only for target-feature facilitation or distractor-feature inhibition. We propose that in singleton search a division of feature space facilitates detection, and that this division works best under conditions of expected repetition. Experiment 5 replicated Experiment 1, but in Experiment 5 we examined response times and long display exposures. Results suggested that previous findings of singleton priming reflect mainly postperceptual factors.
\end{abstract}

Visual search is a key feature of many commonplace human activities, ranging from finding pebbles hidden among batches of beans to spotting weapons in airline baggage screening. A better understanding of visual search, and especially of how experience affects visual search, should help with optimizing training and other practical applications. In the present study, we ask about short-term plasticity in visual search, focusing on two questions: First, how is search processing affected by knowledge of the features of the to-be-detected object? Second, how is search processing affected by events on preceding trials?

In this research, we used an orientation singleton detection task. This kind of search task requires subjects to make forced choice judgments about the location of an element (left vs. right half of the display) that is unique in a given feature. Singleton detection is a relatively simple task and is therefore a good starting point for the questions we want to ask. Singleton detection is also a theoretically important task likely to reveal important aspects of basic attentional and perceptual limitations and mechanisms (Bacon \& Egeth, 1994; Pashler, 1988; Wolfe, 1998).

We begin by defining two distinct types of experiencebased modulation.

The research was supported by Grant MH45584 from the National Institute of Mental Health to H.P. We are grateful to Tom Carr, Brad Gibson, Todd Horowitz, and one anonymous reviewer for useful comments on an early version of this article, and to Kristen Sakamoto and Noriko Coburn for help during all stages of the study. Correspondence relating to this article may be sent to H. Pashler, Department of Psychology 0109, University of California-San Diego, La Jolla, CA 92093 (e-mail: hpashler@ucsd.edu).

\section{Expectation and Repetition Effects}

What we will term the expectation effect refers to an effect produced by knowledge about what will happen next. It is sometimes termed "top-down control." The repetition effect is produced by the sharing of some property between the currently presented stimulus and preceding stimuli; it is sometimes termed "bottom-up priming." These two effects are logically orthogonal, but they are easily confounded (Kristjansson, Wang, \& Nakayama, 2002).

An example of a situation in which both expectation and repetition hold is the fixed occurrence of one feature throughout a whole block. Here, the subject knows definitely what will occur, and the same event also occurred in the previous trial. An example of expectation without repetition is the regular alternation of two feature settings in one block. Here, the subject knows definitely what will occur next, but it is always different from the previous trial. An instance of repetition without expectation may be found in the random distribution of two feature settings in one block: The subject has no knowledge of what will occur, but in some trials the feature happens to repeat that of the previous trial. ${ }^{1}$ Random distribution of feature settings also provides an instance of neither expectation nor repetition: The subject does not know what will occur, and in some trials the feature happens to be different from that of the previous trial.

In the present study, expectation and repetition refer specifically to the expectation and repetition of target/ distractor feature values on the defining feature (i.e., the feature wherein the singleton is unique). Some other studies on repetition and/or expectation have posed questions relating to the response (which we will term the report feature). In the experiments described here, the report feature (location) was always randomly and indepen- 
dently assigned, and variation in this feature merely provided a method of measuring detection.

\section{Perceptual Versus Postperceptual Processing}

In previous work on repetition priming as it affects singleton search, Maljkovic and Nakayama (1994) had subjects search for a featural singleton that could be defined in either of two ways - for example, a white target among black distractors, or a black target among white distractors. They discovered that even when repetition of the feature value was no more likely than alternation, repetition nonetheless sped subjects' responses. That is to say, if in one trial the target was white, in the next trial a white target had an advantage over a black target, even if they were both equally likely to occur. Maljkovic and Nakayama reported that this apparently automatic priming effect was induced only by repetition and was hardly affected by expectation; whether or not the subject had explicit knowledge about the feature of incoming trials had almost no impact on the priming effect. However, their study focused entirely on priming effects in response time (RT) to sustained displays, rather than on accuracy of perception of brief displays; thus, a question remained as to whether the effect they reported arose from changes in perceptual or postperceptual processing stages (see Huang, Holcombe, \& Pashler, 2004, for a discussion suggesting a decisional locus).

To examine this remaining question, we focused in the work reported below on effects observed for response accuracy with brief displays (Experiments 1-4), and in a final experiment, we compared accuracy effects with findings observed for RTs with unlimited viewing time. As has frequently been noted (e.g., Pashler, 1989; Santee \& Egeth, 1982), RT indexes both perceptual and postperceptual stages, whereas accuracy using very brief displays measures only perceptual processing stages. In the present study, our goal was to uncover effects within the perceptual stage, and thus most of our experiments served to assess subjects' accuracy of perception of brief displays.

An instructive recent example of the way in which RT and accuracy measurements differ is provided by Prinzmetal, McCool, and Park (2003), who examined the Stroop effect. This effect occurs with the naming of colors: Usually, when subjects are required to respond to the color of words that are themselves the names of colors, the words' meanings significantly interfere with the naming of their colors, and the naming is substantially slower than it is in a neutral condition in which the words are not related to colors. However, when Prinzmetal et al. performed the same experiment with very brief displays to test the accuracy of color naming, they found a negligible difference between color words and noncolor words. This result suggests that the interference found in RT measurements - the Stroop effect-is postperceptual.

\section{GENERAL METHOD}

\section{Subjects}

Fifty undergraduates from the University of California, San Diego, received credit in a psychology course for their participa- tion in this project. Ten of these subjects took part in each of Experiments $1-5$.

\section{Apparatus}

Stimuli were presented on a 1,024 × 768 MAG DX-15T color monitor driven by an Intel Pentium IV 1.8-G computer. The subjects viewed the displays from a distance of about $60 \mathrm{~cm}$ and entered responses using the keyboard. The program was written in Microsoft Visual Basic 6.0 and was run on Microsoft Windows 98, second edition, using timing routines that were tested using a digital timer.

\section{Stimuli}

Each search display contained 20 lines. Each line was $1.25^{\circ}$ long and $0.21^{\circ}$ wide. All lines were white (luminance $>30 \mathrm{~cd} / \mathrm{m}^{2}$ ) against a black background (luminance $<0.2 \mathrm{~cd} / \mathrm{m}^{2}$ ). All of the lines except one had one particular orientation on the screen (distractors), and the remaining line (the target) had a different orientation. The orientations of the distractors and target for each experiment are described below. Ten lines were randomly placed in each of two $6.65^{\circ}$ (wide) by $14.36^{\circ}$ (high) regions. The regions were located on the left and right halves of the display, each spaced $2.13^{\circ}$ from the center of the screen. The location of the target, in the left or the right half of the display, determined the correct response.

In Experiments 1-4, the display was masked after a brief stimulus duration. The mask was created by presenting two white lines in every possible line location, one with the target orientation and one with the distractor orientation. Stimulus duration was adjusted for each subject to keep accuracy within an appropriate range (.65-.85), with the constraint that for any particular subject, both types of block (defined in each experiment) had the same stimulus duration. (Mean stimulus durations: Experiment 1: $127 \mathrm{msec}$; Experiment 2: $124 \mathrm{msec}$; Experiment 3: $92 \mathrm{msec}$; Experiment 4: $61 \mathrm{msec}$ ). In Experiment 5 , the displays were not masked, but rather remained in view until the subjects responded.

\section{Procedure}

The subjects were told to respond as accurately as possible in Experiments 1-4 and to respond as quickly and accurately as possible in Experiment 5. Each trial began with a small green fixation cross presented for $400 \mathrm{msec}$ in the center of the screen. After a short blank interval (400 msec), the display appeared. The subjects were instructed to fixate on the cross and subsequently to search for the differently oriented line in each display. In Experiments 1-4, each display was masked after a short stimulus duration. In Experiment 5 , the displays remained until the subjects responded. The subjects decided whether the target was on the left or the right and responded by pressing one of two adjacent keys with fingers of the right hand (" $\mathrm{j}$ " for left, " $\mathrm{k}$ " for right). A tone sounded for about $500 \mathrm{msec}$ to indicate whether the response was correct, and the next trial began $400 \mathrm{msec}$ later. Each subject performed 10 blocks of 100 trials each, with the first 2 blocks excluded as practice. Different block conditions (as described below for each experiment) alternated for each subject and were counterbalanced across subjects.

\section{EXPERIMENT 1}

In Experiment 1, the task was to find and report the location of a vertical target line among horizontal distractor lines or of a horizontal target among vertical distractors. There were two, alternating types of block: In a homogeneous block, the feature settings (horizontal or vertical orientation) of the target and distractors were the same in every trial. Thus, in a homogeneous block, one might have all vertical distractors and all horizontal targets, or vice versa. In a random block, the feature settings were selected randomly for each trial. The feature 
settings of homogeneous blocks alternated and were counterbalanced across subjects. An example of the sequence of block settings experienced by the subjects would be homogeneous $\mathrm{F}$ (eature setting)1, random, homogeneous F2, random, homogeneous F1, random, homogeneous F2, random, homogeneous F1, random.

\section{Results and Discussion}

The results of Experiment 1 (as well as those of Experiments 2-4) are presented in the top panel of Figure 1. The term repeated trials in random blocks refers to data from random blocks for which the target feature value (e.g., vertical) was the same as in the preceding trial. The term alternated trials in random blocks refers to data from random blocks for which the target feature value (e.g., vertical) differed from that of the target shown in the preceding trial. In this experiment, accuracy was higher in homogeneous blocks than in random blocks, but there was no apparent difference between repeated and alternated target-feature trials. The effects are computed in the bottom panel of Figure 1.

The results of Experiment 1 indicate that repetition and expectation of feature settings (both operative in homogeneous blocks) are jointly sufficient to produce a significant perceptual advantage. However, repetition alone (in conditions in which the subjects had no basis for expecting what the feature of the target would be) produced very little effect. There are two plausible interpretations of this result: Either expectation has a significant effect, whereas repetition has none, or alternatively, an effect occurs only when repetition and expectation are simultaneously present. To assess these two explanations, we needed to examine what happens when the target feature is expected but not repeated.

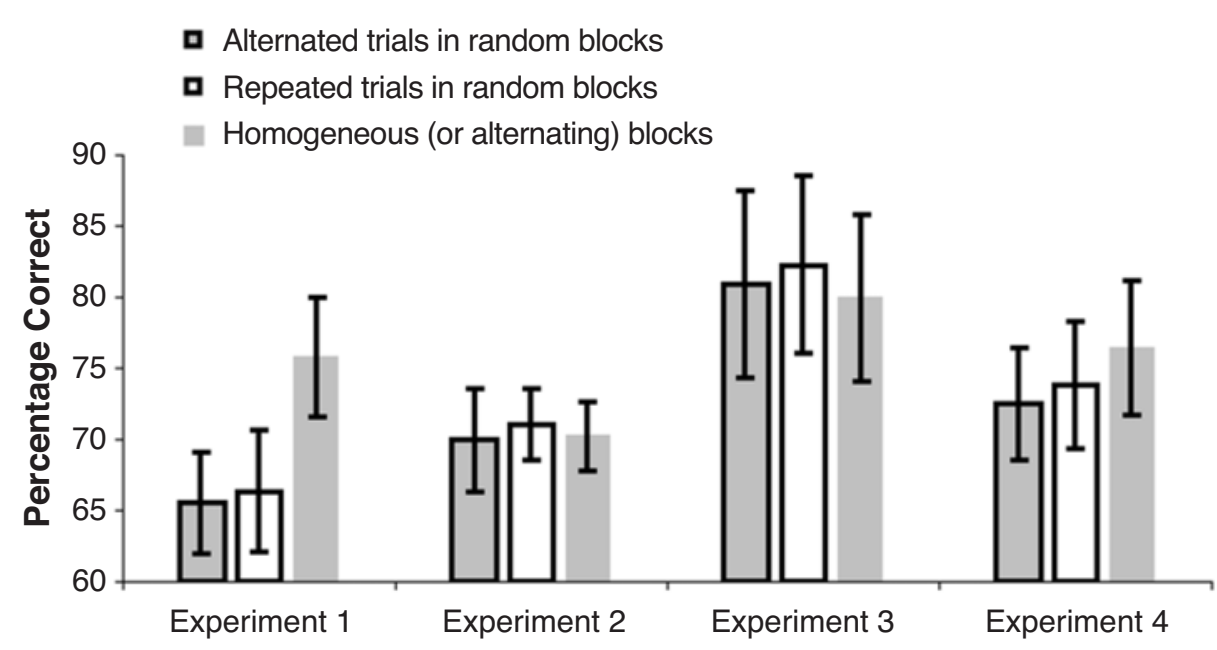

Overall advantage of homogeneous (or alternating) blocks

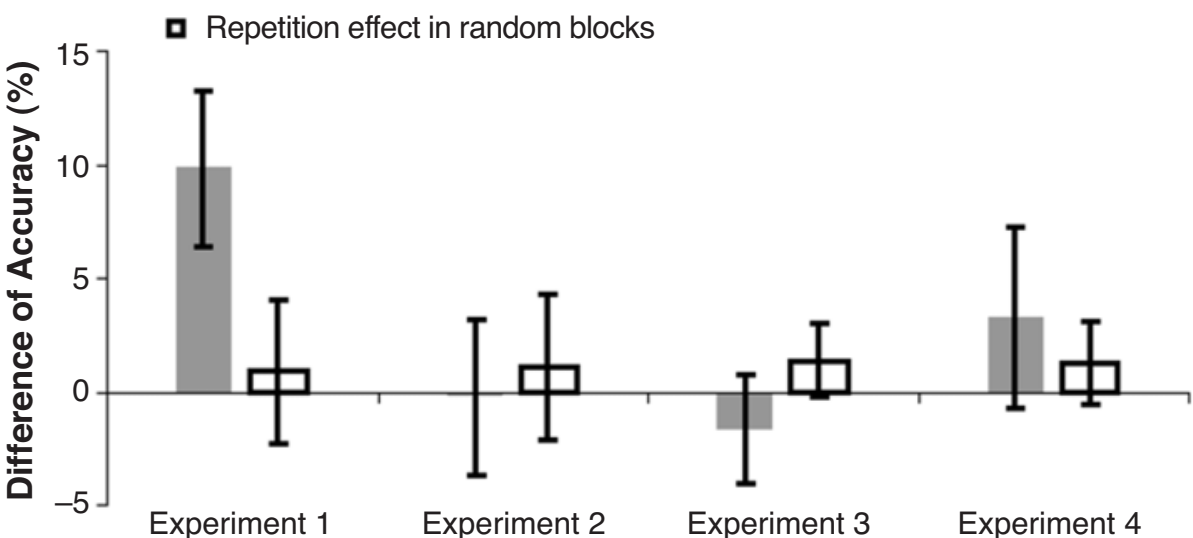

Figure 1. Results of Experiments 1-4. Top panel: Accuracy for all conditions. Bottom panel: Difference between homogeneous (or alternating) blocks and random blocks. Error bars indicate confidence interval $(p<.05)$. This difference is significant in Experiment 1 but not in Experiments 2-4. The repetition effect in random blocks is not significant in any of Experiments 1-4. (Mean stimulus durations: Experiment 1, 127 msec; Experiment 2, 124 msec; Experiment 3, 92 msec; Experiment 4, 61 msec.) 


\section{EXPERIMENT 2}

Experiment 2 was designed to arrange conditions so that subjects would have an expectation for a target feature value, but that value would not be repeated. As in Experiment 1, the subjects' task was to find and report the location of a vertical target among horizontal distractors or of a horizontal target among vertical distractors. Once again, there were two alternating types of block: In an alternation block, the feature settings in each trial were reversed from those of the previous trial; this kind of "predictable alternation" has been used previously by Maljkovic and Nakayama (1994) to measure the role of expectation. In a random block, the feature settings were decided randomly for each trial.

\section{Results and Discussion}

The results of Experiment 2 are given in the top panel of Figure 1. Accuracy in alternated blocks was not higher than it was in random blocks. In random blocks, there was no apparent difference between repeated trials and alternated trials. The effects are computed in the bottom panel of Figure 1.

Of the two possible interpretations of Experiment 1 that were discussed above, Experiment 2 clearly favors the latter: that a significant perceptual advantage is to be gained only by the joint operation of both expectation and repetition. For this reason, hereafter we will call this priming effect the expectation-repetition effect. An acrossexperiments analysis of variance (ANOVA) indicated a significant interaction $[F(1,18)=9.79, p<.01]$ between expectation and repetition. The absence of any repetition effect in the random blocks of this experiment confirmed the similar result of Experiment 1.

In Experiments 3 and 4, we asked another question about the expectation-repetition effect: Is it due to inhibition of the feature value of the distractor or to facilitation for detection of the target feature? In Experiments 1 and 2 , these factors were confounded, because when a target feature value was repeated and expected, the feature value for the distractor was also repeated and expected.

\section{EXPERIMENTS 3 AND 4}

In Experiment 3, the task was to find and report the location of a diagonal target $\left(45^{\circ}\right.$ left tilted) among vertical or horizontal distractors. In a homogeneous block, the distractors' orientation (either vertical or horizontal) was the same in every trial. In a random block, the distractors' orientation was determined randomly for each trial. In both types of block, the target's orientation was always the same. If the expectation-repetition effect is due to the inhibition of distractor features, in Experiment 3 we should see a significant advantage of homogeneous over random blocks, since the distractor feature values were repeated predictably in homogeneous blocks and varied randomly in random blocks.

In Experiment 4, the task was to find a vertical or horizontal target among diagonal distractors. In a homoge- neous block, the target's orientation (vertical or horizontal) was the same in every trial. In a random block, the target's orientation was determined randomly for each trial. In both types of block, the distractors' orientation was always the same ( $45^{\circ}$ left tilted). If the expectationrepetition effect is due to the facilitation of the target feature, in Experiment 4 we should see a significant advantage of homogeneous over random blocks, since the target feature values were repeated predictably in homogeneous blocks and varied randomly in random blocks.

\section{Results and Discussion}

The results of Experiments 3 and 4 are presented in the top panel of Figure 1. In both experiments, the accuracy of homogeneous blocks was not significantly higher than that of random blocks. In random blocks, there was no apparent difference between repeated trials and alternated trials. The effects are computed in the bottom panel of Figure 1.

The results of Experiment 3 suggest that there is no distractor-feature inhibition effect. The results of Experiment 4 indicate only a small target-feature facilitation effect. The latter approaches significance, however, and should therefore be acknowledged as a potentially real effect. Nevertheless, it is considerably smaller than the expectation-repetition effect identified in Experiment 1 [interaction in across-experiments ANOVA: $F(1,18)=$ $6.12, p<.05]$. The expectation-repetition effect cannot be explained by either distractor-feature inhibition or targetfeature facilitation, nor by their algebraic summation. Evidently, the effect relies on some interaction between processing of the distractor and the target features. We discuss this point further in the General Discussion section.

\section{EXPERIMENT 5}

Our conclusions from Experiments 1-4 differ from those described by Maljkovic and Nakayama (1994). As mentioned above, we believed that the measurement of accuracy in processing of very brief displays (such as those used in the studies reported above) reveals the character of perceptual-level effects, whereas RT measurements (as in Maljkovic and Nakayama's work) are likely to include contamination from postperceptual effects as well. Alternatively, however, the differences between our results and theirs could reflect other differences in methodology.

The purpose of Experiment 5 was to determine whether the critical difference between our Experiment 1 and the research of Maljkovic and Nakayama (1994) was the use of brief displays with accuracy measures. Experiment 5 was identical in method to Experiment 1, except that the displays remained present (and unmasked) until subjects responded, and RTs were measured instead of accuracy.

\section{Results and Discussion}

In Experiment 5, the mean RTs were the following: for homogeneous blocks, $480 \mathrm{msec}$ (error rate $=2.7 \%$ ); for repeated trials in random blocks, $548 \mathrm{msec}$ (error rate = $1.8 \%$ ); and for alternated trials in random blocks, $594 \mathrm{msec}$ 
(error rate $=2.8 \%$ ). Responses in homogeneous blocks were significantly faster than those in random blocks $[$ difference $=91 \mathrm{msec} ; F(1,9)=51.23, p<.001$ ]; in random blocks, repeated trials were significantly faster than alternated trials [effect $=46 \mathrm{msec} ; F(1,9)=9.62, p<.02$ ]

The results of Experiment 5 closely resemble those of Maljkovic and Nakayama (1994), confirming that the effects they found can be safely compared with those in our Experiment 1. There is an important difference between our Experiments 1 and 5: Repetition in random blocks produced no obvious effect on accuracy in Experiment 1, whereas it produced a significant effect on RTs in Experiment 5. It seems unlikely that the substantial 46-msec effect in Experiment 5 would reflect an equivalent difference in perceptual processing, since the effect on accuracy was a mere $0.87 \%$ in Experiment 1 . Also, the fact that in Experiment 1 accuracy reached $71 \%$ at the stimulus duration of $127 \mathrm{msec}$ excludes the possibility that accuracy generally increases very slowly over time. Note as well that the accuracy of $71 \%$ should mean that accuracy was near its maximum level of sensitivity, well clear of floor or ceiling effects. That is to say, unexpected repetition can make responses significantly faster, but for a brief display, it does not make them more accurate. It seems, therefore, that the repetition effect on speed reflects a different underlying cause from our perceptual expectation-repetition effect of Experiment 1 . The former presumably arises exclusively in some postperceptual stages (attentional or decisional), whereas the latter arises in perceptual processing operations. In addition, Maljkovic and Nakayama (1994) suggested that their priming effect is induced mainly by repetition, but our expectation-repetition effect seems to be induced in no small part by expectation as well.

\section{GENERAL DISCUSSION}

\section{Cumulative Repetition Effect}

Subjects searched displays for a line that differed in orientation from the other lines in a display. In the random blocks of Experiment 1 (in which the orientation of target lines varied randomly from trial to trial), repetition of the orientation of the target line by itself did not improve accuracy of judgments. We therefore concluded that expectation is a necessary component of the effect observed in homogeneous blocks. Our conclusion might be open to this objection: Perhaps each singleton repetition produces only a very small effect, but these effects accumulate across several successive repetitions, resulting in a cumulative homogeneous-block effect. In this way, the effect could be explained without the necessity of expectation. Such a notion is not implausible, since Maljkovic and Nakayama (1994) reported that their repetition effect did accumulate over successive repetitions.

Reanalyzing the data from random blocks in our Experiments 1 and 2 (which were identical for random blocks), we plotted the repetition effect as a function of number of successive repetitions of the target feature value. As is shown in Figure 2, we found no significant accumulation. We did not analyze runs of more than four successive repetitions, since those trials were very rare and did not allow a good estimation of the repetition effect. Those trials are also unlikely to be important in this connection, since Maljkovic and Nakayama (1994) reported that in their experiment the first four successive repetitions produced an effect only slightly smaller than the one for very many successive repetitions. It is safe to say that accumulation of successive repetitions cannot explain the expectation-repetition effect in Experiment 1; expectation, therefore, must be a component of our explanation.

We do not mean to say, by the analysis above, that a long queue of successive repetitions would not produce an effect without explicitly instructed expectation. Even if subjects are instructed that feature settings will be random, when many repetitions occur successively, the subjects will begin to realize (consciously or unconsciously) that the instruction was wrong and to expect the repeated feature (Lindman \& Edwards, 1961). What does at least seem clear from the analysis above is that accidental repetition in several successive trials of a random block does not produce an advantage comparable to that enjoyed in homogeneous blocks.

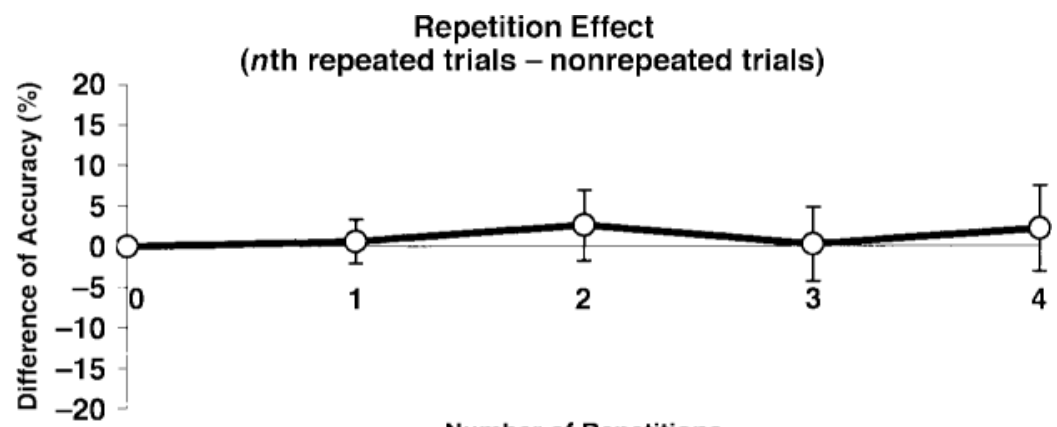

Number of Repetitions

Figure 2: Cumulative effect of successive repetitions in the random blocks of Experiments 1 and 2 . There is no apparent accumulation. 


\section{A Feature Divider Account}

Although the existing results are probably not sufficient to reveal the nature of the expectation-repetition effect fully, we propose a tentative account. This account relies on the notion of what we will term a feature divider in featural singleton detection. The feature divider is a mechanism that implements a categorization rule that divides orientation feature space into two parts. As a result of the feature divider's operations, elements with some set of orientations are "highlighted," whereas the remainder are ignored. In each of our trials, the feature divider had to find a parameter that could successfully highlight only one item while it ignored the others. In Experiment 1, the feature divider could use the same parameter (e.g., a parameter highlighting the vertical and ignoring the horizontal) for all trials of a homogeneous block. During random blocks, however, it had to switch parameters back and forth. For example, in one trial it used a parameter selecting the vertical lines, but if in the next trial "vertical" became the distractor feature that was to be ignored, the feature divider had to use a new parameter. This account suffices to explain the difference in subjects' performance between homogeneous and random blocks. In Experiments 3 and 4, a single parameter could be used for all trials of a random blocka parameter that distinguished both vertical and horizontal lines from $45^{\circ}$ left-tilted lines-so there was little advantage in homogeneous blocks. The feature divider concept has been previously demonstrated in the dimension of color (D'Zmura, 1991). It seems natural to assume that it should apply broadly for all feature dimensions in feature processing.

Some readers, noticing that the mean stimulus duration was longer in Experiment 1 than in Experiments 3 and 4, may object that it was only this difference of duration that brought about the different results; Experiment 1 , they may say, allowed more time for effects to become manifest. We believe that this account, though not impossible, is unlikely. All that should matter for the manifestation of the effects under discussion is the quantity of information exposed to the subjects. Although stimulus durations were shorter in Experiments 3 and 4, accuracy was higher-indicating that subjects actually received more information.

We believe that the experiments' different stimulus durations reflect the relative difficulty of the tasks. The difference in duration is not the reason behind, but rather the result of, the expectation-repetition effect. This premise allows us to address another question: The results of Experiment 1 indicate that some advantage available in homogeneous blocks was absent from random blocks. Was the lack of this difference in Experiments 3 and 4 due to the advantage's being always available, in both homogeneous and random blocks, or rather to its never being available? Since the tasks in Experiments 3 and 4 were easier as a whole, it seems that the answer must be always: The advantage was always available, even in random blocks. This conclusion is consistent with the feature divider account.
Having explained why the expectation-repetition effect showed up in Experiment 1 but not in Experiments 3 and 4, we face another question: Why is this effect not activated by repetition alone? If it were an attribute of the feature divider that it preserved the parameter of each trial just past, then accidental repetition in Experiment 1 random blocks should have created a significant advantage over alternation. Having observed no such advantage, we assume that, perhaps because the consequence of a wrong parameter may be very high, the feature divider does not use the parameter of the last trial when the next trial is not predictable. Instead, for each trial, the divider prescreens the new display to set up the appropriate parameter. This view can be put in another way: The underlying mechanism can choose to work in one of two ways - either preparing for a certain feature or not. This account fits well with previous work that found a distinction between "detecting a feature" and "detecting a singleton" (Bacon \& Egeth, 1994). We must then ask why, in Experiment 2, there was no advantage for predicted alternation over random blocks. Perhaps a feature divider can only hold one parameter at a time, so that replacing the old parameter with a new one always results in the loss of the old. This would be entirely natural if the parameter reflected some property or state of the feature divider itself. Even if the target in the next trial can be predicted with certainty, the feature divider cannot skip the prescreening - it is necessary for the establishment of the appropriate parameter.

There are, of course, alternative accounts of the expectation-repetition effect. One account deserving some attention holds that the effect results from negative priming (Tipper, 1985) - the disadvantage of attending to an object or feature that was previously inhibited. A direct test to distinguish and decide between this account and the feature divider account would be to imagine three feature values in orientation space, carefully selected so that the target orientation (O1) falls in the middle of a line connecting two possible distractor orientations (O2 and $\mathrm{O} 3)$. The feature divider account predicts a significant advantage for blocks in which only $\mathrm{O} 2$ or only $\mathrm{O} 3$ is used over blocks in which $\mathrm{O} 2$ and $\mathrm{O} 3$ are randomly mixed. The negative-priming account predicts no such advantage. Unfortunately, such a direct test is not possible until we have an understanding of the organization of orientation space as explicit as our understanding of color space.

At present, we can offer two arguments for the selection of the feature divider over the negative-priming account: First, as was discussed above, expectation is an important component of the expectation-repetition effect. It is not implausible that a feature divider would be significantly affected by voluntary control, as in the act of conscious expectation. However, voluntary control plays little part in negative priming; the negative priming of a certain feature will not stop simply because you are not expecting it (Tipper, 1985). Second, it is believed that negative priming reflects postperceptual factors (Neill, 1997); thus, it should not show up in a measurement of 
perceptual accuracy of brief displays. Future research is needed to test these and other possible accounts further.

\section{Implications for Other Feature-Based Attention Studies}

The two questions we raised at the beginning of this article are very broad, and we have only tested one of the simplest related conditions. Studies of what is usually called feature-based attention also bear directly on these questions (Farell \& Pelli, 1993; Moore \& Egeth, 1998; Shih \& Sperling, 1996). In these studies, "attending to one feature" usually implies the application to a feature of both expectation and repetition. Results suggest that feature knowledge-knowledge, for example, that the color of a search object is red-does not improve the perception of red objects. It may help only by giving red objects search priority over other objects. In the most recent of these studies, Moore and Egeth (1998) presented evidence from parallel experiments in which measurements of RT were compared with measurements of perceptual accuracy. In their experiment, knowledge of feature settings increased the speed of responses to sustained displays but did not increase the accuracy of responses to very brief displays.

A compelling conclusion may be drawn from Moore and Egeth (1998) and the other studies mentioned above: Feature information does not result in an immediate improvement to perception of a specific feature. There are various possible accounts for this phenomenon. The current interpretation of the authors cited is that feature information can affect visual processing only by guiding spatial attention. To put this idea another way, spatial attention mediates feature-based attention. Location is the "master map" of visual attention: Search operations on a certain feature are facilitated by, and performed subsequently to, the highlighting of all locations containing that feature. This notion is widely expressed in attention models (Cave, 1999; Wolfe, Cave, \& Franzel, 1989) and has been supported experimentally (Johnston \& Pashler, 1990).

At first glance, the present work appears to contradict these feature-based attention studies: Farell and Pelli (1993), Shih and Sperling (1996), and Moore and Egeth (1998) all suggest that feature information cannot affect perception in brief displays, but the present study shows just the opposite. However, further analysis of their rationale, and of the distinctions between their methods and ours, indicates that our result does not in fact undermine, but rather strengthens, their notion of "spatial mediation of color-based attention."

According to the spatial mediation rationale of featurebased attention, the results of Moore and Egeth (1998) should be interpreted thus: Knowledge about a target feature must be translated into location information, which is then used to start the attentional processing of other search dimensions (e.g., character identity). In experiments for which the display time is very brief, this whole process takes too long to manifest any advantage gained from feature knowledge. If this account is true, then the said advantage may become evident in a task that requires the subject to report only location information; the second step is not required, so processing can be finished in a much shorter period.

An important distinction between the present work and the three studies mentioned is that in the latter studies, subjects were required to report secondary features, whereas we required ours to report only location. Our respective results confirm the rationale above. In terms of the feature divider account, the feature divider is the tool that creates the master map of location. In a study such as Moore and Egeth (1998), the location map produced by the feature divider can be used by selective attention, but because this usage is slow, the effect of the feature divider cannot be observed. In the present study, the location map produced by the feature divider was itself the desired result, so the feature divider's effect was observed directly.

Even if the present work provides clear support, as suggested above, for the spatial mediation explanation of color-based attention, there are alternative possibilities. The present study also differs from previous studies (Farell \& Pelli, 1993; Moore \& Egeth, 1998; Shih \& Sperling, 1996) in several respects other than the one we have discussed. One of these is the relative difficulty of our subjects' task; might it be that expectation and repetition carry an advantage only for easy tasks? Further research is needed to explain why an expectation-repetition effect shows up in our study but not in theirs, and thus to determine how feature-based attention affects perception.

\section{How Is the Perceptual Stage Affected?}

When we insist that the expectation-repetition effect occurs during the perceptual stage, we do not mean that the perception of an object is changed or that an object is perceived, as it were, as something other than itself. Prinzmetal, Amiri, Allen, and Edwards (1998) demonstrated that attention only reduces the variability of observed feature values and does not change the observed feature's mean value. That is to say, paying attention to a red object will not make it redder or brighter, but will only make the redness more precise and the related judgment more accurate. Our case is probably the same: Expectation and repetition only help us get more precise information about the orientation singleton; the orientation itself of course will not, because of expectation or repetition, be perceived as altered. This view is consistent with a feature divider account holding that what changes in perception is the efficiency of information extraction, not the information itself.

\section{CONCLUSION}

The five experiments reported here indicate several things:

1. The perception of feature cues can be improved by priming. This priming effect is not triggered by either 
unexpected repetition or expected alternation alone, but only by expected repetition. Inconsistency between this expectation-repetition effect and previous observations of no effect of feature-based attention on perception is probably due to differences in the previous studies' respective tasks: A feature-based attention effect can be observed when subjects are required to report location alone, because the effect depends on "spatial mediation."

2. This perceptual improvement cannot be fully explained by either target-feature enhancement or distractorfeature inhibition, nor by their summation. More complicated interactive processing must be involved.

3. The expectation-repetition effect reported here is different from the repetition priming effect (Maljkovic \& Nakayama, 1994). The latter depends merely upon repetition, not expectation, and seems to be exclusively postperceptual; the former requires both repetition and expectation and seems to have perceptual components.

To account for these findings, we proposed a tentative feature divider account.

\section{REFERENCES}

BACON, W. F., \& Egeth, H. E. (1994). Overriding stimulus-driven attentional capture. Perception \& Psychophysics, 55, 485-496.

CAve, K. R. (1999). The feature gate model of visual selection. Psychological Research, 62, 182-194.

D'ZMura, M. (1991). Color in visual search. Vision Research, 31, 951966.

Farell, B., \& Pelli, D. G. (1993). Can we attend to large and small at the same time? Vision Research, 33, 2757-2772.

Huang, L., Holcombe, A. O., \& PASHLer, H. (2004). Repetition priming in visual search: Episodic retrieval, not feature priming. Memory \& Cognition, 32, 12-20.

Johnston, J. C., \& PASHLER, H. (1990). Close binding of identity and location in visual feature perception. Journal of Experimental Psychology: Human Perception \& Performance, 16, 843-856.

KRISTJANSSON, A., WANG, D., \& NAKAYAMA, K. (2002). The role of priming in conjunctive visual search. Cognition, $\mathbf{8 5}, 37-52$.

LiNDMAN, H., \& EDWARDS, W. (1961). Supplementary report: Unlearning the gambler's fallacy. Journal of Experimental Psychology, 62, 630.

MaljKovic, V., \& Nakayama, K. (1994). Priming of pop-out: I. Role of features. Memory \& Cognition, 22, 657-672.
Moore, C. M., \& EgETh, H. (1998). How does feature-based attention affect visual processing? Journal of Experimental Psychology: Human Perception \& Performance, 24, 1296-1310.

NEILL, W. T. (1997). Episodic retrieval in negative priming and repetition priming. Journal of Experimental Psychology: Learning, Memory, \& Cognition, 23, 1291-1305.

PASHLER, H. (1988). Cross-dimensional interaction and texture segregation. Perception \& Psychophysics, 43, 307-318.

Pashler, H. (1989). Dissociations and dependencies between speed and accuracy: Evidence for a two-component theory of divided attention in simple tasks. Cognitive Psychology, 21, 469-514.

Prinzmetal, W., Amiri, H., Allen, K., \& EdWARds, T. (1998). The phenomenology of attention: 1 . Color, location, orientation, and "clarity." Journal of Experimental Psychology: Human Perception \& Performance, 24, 261-282.

Prinzmetal, W., McCoOL, C., \& PARK, S. (2003). Attention: Reaction time and accuracy reveal different mechanisms. Manuscript submitted for publication.

Santee, J. L., \& Egeth, H. E. (1982). Do reaction time and accuracy measure the same aspects of letter recognition? Journal of Experimental Psychology: Human Perception \& Performance, 8, 489-501.

ShIH, S., \& SperLing, G. (1996). Is there feature-based attentional selection in visual search? Journal of Experimental Psychology: Human Perception \& Performance, 22, 758-779.

TIPPER, S. P. (1985). The negative priming effect: Inhibitory priming by ignored objects. Quarterly Journal of Experimental Psychology, 37A, 571-590.

Wolfe, J. M. (1998). Visual search. In H. Pashler (Ed.), Attention (pp. 13-74). London: Psychology Press.

Wolfe, J. M., Cave, K. R., \& Franzel, S. (1989). Guided search: An alternative to the feature integration model for visual search. Journal of Experimental Psychology: Human Perception \& Performance, $\underline{\mathbf{1 5}_{2}}$ $\underline{419-433 .}$

\section{NOTE}

1. The subject may have random expectations from trial to trial (Lindman \& Edwards, 1961). For example, even if there is no reason to believe the next trial will be white or black, a subject may still expect it to be one or the other. However, there should not be any consistent expectation across the whole block, and those random expectation effects should cancel each other out.

(Manuscript received June 9, 2003; revision accepted for publication April 9, 2004.) 